\title{
External Quality Assessment of Molecular Biology-Based Methods Used in Laboratories of Clinical Chemistry and Human Genetics
}

\author{
Andreas Braun ${ }^{1,2}$, Thomas Deufel ${ }^{3}$, Wolf-J ochen \\ Geilenkeuser ${ }^{4}$, Michael Neumaier ${ }^{5}$, Gerhard Röhle ${ }^{4}$, \\ Adelbert Roscher ${ }^{1}$ and Christoph Wagener ${ }^{5}$
}

\author{
${ }^{1}$ Abteilung für Klinische Chemie und Biochemie, Dr. von \\ Haunersches Kinderspital, Ludwigs-Maximilians-Universität \\ München, München, Germany \\ 2 Sequenom Instruments GmbH, Hamburg, Germany \\ ${ }^{3}$ Institut für Klinische Chemie und Laboratoriumsdiagnostik, \\ Friedrich Schiller-Universität, J ena, Germany \\ ${ }^{4}$ Referenzinstitut für Bioanalytik, Deutsche Gesellschaft für \\ Klinische Chemie, Bonn, Germany \\ ${ }^{5}$ Abteilung für Klinische Chemie, Universitätskrankenhaus \\ Eppendorf, Hamburg, Germany
}

The Reference Institute of Bioanalysis of the German Society of Clinical Chemistry has performed the first external assessment of molecular genetics methods used in medical diagnosis. The following procedures were tested: (I) DNA preparation from whole blood, (II) PCR amplification using "standard" primers, and (III) submarine agarose gel electrophoresis. Out of 50 participants, 45 returned samples for evaluation.

Key words: External quality assessment; Molecular biology; PCR; DNA preparation; DNA electrophoresis.

\section{Introduction}

During recent years, molecular genetic methods have shown great potential in medical diagnostics. The spectrum of techniques includes the detection of polymorphic sites and somatic and germ line mutations as well as identification of tumour-specific transcripts and microbial and viral genomes. Because of the multitude of different applications it is still difficult to perform a specific external quality control for every procedure. Therefore, the Reference Institute of Bioanalysis of the German Society of Clinical Chemistry in cooperation with the Society's working group on "Molecular Biology Methods in Clinical Chemistry" attempted to address the issue of quality assessment with a pilot study to assess individual analytical steps in the complex molecular diagnostic process using genomic DNA as template. The PCR samples returned to the institute were jointly evaluated in order to achieve better comparability of the results obtained in various laboratories.

\author{
Participants and Control Materials
}

Participants in this external quality assessment trial for molecular biology diagnostics came from institutes of human genetics, medical genetics, and clinical chemistry as well as from private medical laboratories. We received submissions from Germany, Austria and Switzerland. Forty-five of the 50 trial samples sent out were returned for joint evaluation.

Citrated blood from a female volunteer (DNA-1), DNA prepared from leukocytes of a male volunteer (DNA-2), and "standard" primers were sent out as control material and diagnostic reagents, respectively. The primer solution consisted of a mixture of 4 oligonucleotides; K366-F: 5'-ATG GAA GTC TAA AAG CCT GG-3'; K366-R: 5'-GAA GAG GTG TाT CCA CAG AG-3'; SX9: 5'-GCA CCT TTC AAT TाT GTC GC-3'; and SX10; 5'-AAG GCC TाT ATT AGC CAG AG-3'. Primers were received from MWG-Biotech, Ebersberg, Germany, and used as supplied. K366 primers amplify exon 4 of the human vitamin Dbinding protein gene located on chromosome 4 (1) and SX primers are specific for the entire coding region of the human SRY gene located on the Y chromosome (2). To choose the optimal PCR programme the participants received information about the median $\mathrm{T}_{\mathrm{m}}$ of all primers used, the optimal $\mathrm{Mg}^{2+}$ concentration, and were told that the expected size of the PCR products was in a range between 300 and 800 base pairs. The PCR products utilizing DNA-1 as template were named P-D1 and using DNA-2 as template were named P-D2.

\section{Results and Interpretation}

\section{Basic evaluation parameters and general comments}

The basis for the evaluation of fragment lengths described by the participants in the assessment trial was the absolute fragment length in base pairs as determined by DNA sequence analysis. For the chromosome 4-specific fragment the length was $366 \mathrm{bp}$, and for the $Y$ chromosome-specific product, $779 \mathrm{bp}$, respectively. Twenty $\mu \mathrm{l}$ of the returned PCR sample were mixed with $5 \mu$ of application buffer (20\% Ficoll 400 in one fold concentrated Tris-Borate-EDTA [1xTBE] including bromphenol blue) for simultaneous electrophoretic separation. Seven $\mu$ of each mixture were analysed using $1 \%$ agarose gels in 1XTBE buffer under identical conditions. One or both of the samples returned by participants no. $17,18,22,23,25,29$, and 39 contained either too little or no material for the joint analysis. After electrophoresis, the ethidium bromide stained gels were digitalised unter UV light using a videosystem (MWG-Biotech). A semi-quantitative examination of the products was carried out by densitometry with RFLP Scan V2.1 (ScanalyticS, Vienna, USA). Amplitudes of absorption were related to the $500 \mathrm{bp}$ band of the DNA length standard ( $1 \mathrm{~kb}$ ladder, Gibco/BRL) applied to all gels in order to compensate for differences in staining and/or digitalisation. 

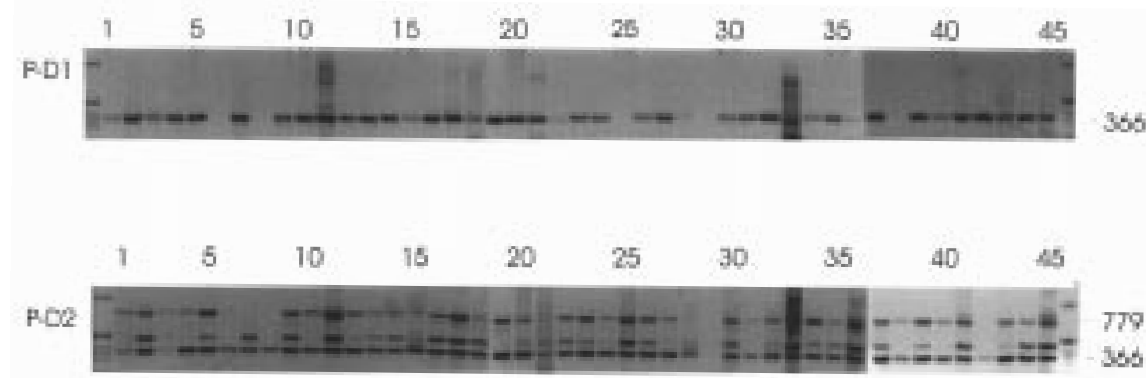

Fig. 1 J oint electrophoretic evaluation of PCR products returned by 45 participants in the programme. The gels consisted of $1 \%$ agarose (UltraPURE, Gibco/BRL) in $1 \times$ TBE.

P-D1: female DNA prepared from whole blood by the partici-

Various methods for DNA preparation, different thermophilic polymerases, PCR cyclers and programmes as well as agarose gel electrophoretic procedures were used as analytical systems. The PCR products of all participants in the trial are presented in Figure 1. The PCR with these "standard" primers permits a sex-specific diagnosis of DNA. In the case of female DNA, a product with a length of $366 \mathrm{bp}$ (P-D1) is anticipated, and two products with a length of $366 \mathrm{bp}$ and $779 \mathrm{bp}$ (P-D2) are expected using male DNA.

The implementation of a polymerase chain reaction not established in a laboratory is certainly difficult. However, since most of the participants obtained largely comparable results, the divergences observed in some cases might allow some conclusions regarding the quality of reagents and analytical systems used. The bands obtained by most participants in the P-D2 reaction with a length of about 500 bp are highly variable in intensity and are seen as strong secondary bands. Analysis of the individual primers using mass spectrometry showed disruptions in the synthesis of primer SX9, which is one of the primers specific for the Y chromosomal 779 bp product (data not shown). This might cause mispriming during PCR using male DNA as template, but other mechanisms such as non-optimised $\mathrm{Mg}^{2+}$ concentration in the buffer system used could also be responsible for this observation. Further strong or weak secondary bands seen by only some participants are most likely attributable to the analytical modules and/or reagents used.

\section{DNA preparation}

The comparison of the densitometric results of the 366 bp product from the P-D1 and P-D2 reactions (Fig. 2) allows certain conclusions regarding the quality of prepared DNA in comparison to the control DNA provided. Twenty-seven samples were evaluated. The criterion for assessment was the clear evidence of the $366 \mathrm{bp}$ bands in both reactions and the $779 \mathrm{bp}$ fragment in reaction P-D2, their easy differentiation from secondary bands and the fact that sufficient material was returned for the joint evaluation. According to these criteria four participants achieved a high quality (quotient $>1.0$ ), nine attained moderate qualities (between 0.7 and 1.0), and the DNA preparation from 14 participants showed pants; P-D2: male control DNA prepared according to ref. 3 were used for PCR amplification. Fragment size in base pairs is indicated at the right margin.

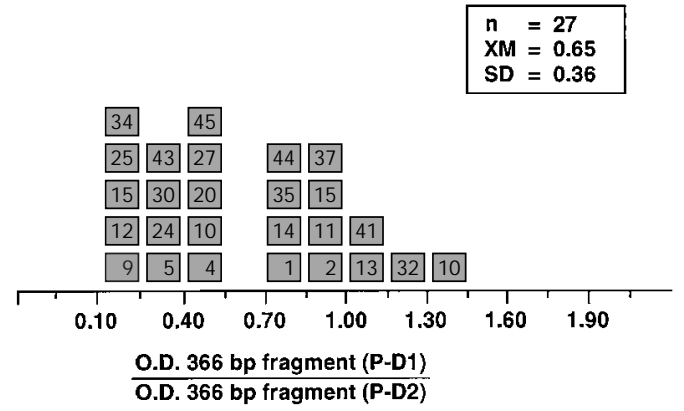

Fig. 2 Ratio of densitometric results of the $366 \mathrm{bp}$ product from P-D1 and P-D2 reactions. Insert shows the statistical analysis of results from 27 participating laboratories.

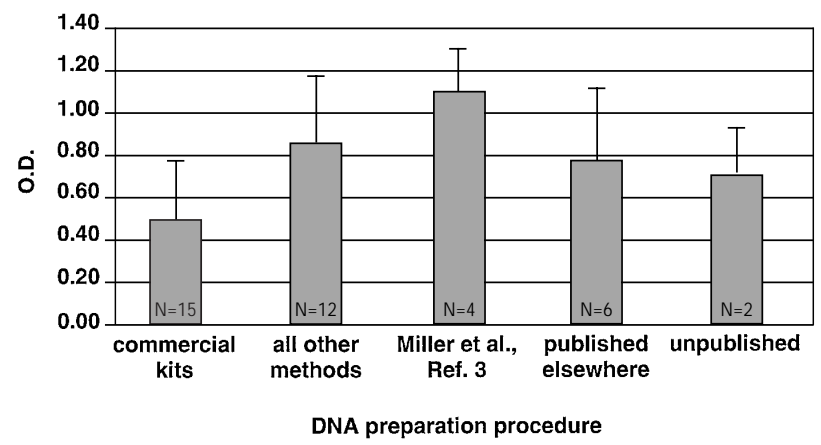

Fig. 3 Densitometric P-D1/P-D2 ratio of the $366 \mathrm{bp}$ products according to the DNA preparation procedures.

a considerably lower quality $(<0.55)$. In 15 of the participants included in this evaluation, the DNA was prepared from whole blood using commercial kits and in 12 the DNA was extracted according to methods published in the literature (3-9) or developed in their own laboratories. The mean quality of DNA prepared by using commercial kits was considerably lower compared to that of all other methods (Fig. 3). A possible reason could be that commercial kits are not optimised to extract high quality DNA suitable for PCR amplification from citrated blood used in this trial. The best quality of DNA was achieved by preparing the DNA according to the method described by Miller et al. (3). That was also the procedure used to prepare the distributed control DNA. 


\section{PCR System}

The densitometric ratio of the $366 \mathrm{bp}$ and $779 \mathrm{bp}$ products of the P-D2 preparation allows some conclusions regarding the PCR system used (Fig. 4). Balanced amplification conditions should produce equal amplification of these two products. Since all participants used the DNA control material for this part of the trial, the amplification reactions comprise the thermophilic polymerase, buffer conditions as well as the instruments used. The returns of 33 participants were evaluated. Inclusion criteria for this evaluation were that both bands were clearly visible without interferences from secondary bands. The mean ratio of the O.D. of the $779 \mathrm{bp}$ fragment to the O.D. of the $366 \mathrm{bp}$ fragment in all samples was 0.43 (SD 0.29). Almost all reactions performed by the participants led to a stronger amplification of the $366 \mathrm{bp}$ product. Thus, for future quality assessment trials, it might be necessary to give additional information on the sequences of primers and the degree of multiplexing of the PCR.

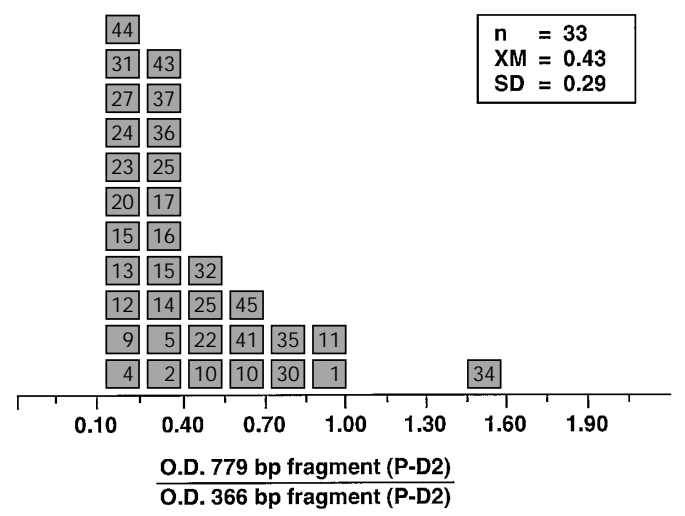

Fig. 4 Ratio of densitometric results of the $366 \mathrm{bp}$ and $779 \mathrm{bp}$ products from P-D2 reactions. Insert shows the analysis of results from 33 participating laboratories.

\section{Fragment length determination}

The fragment lengths stated by the individual participants in the trial are presented in Figure 5 ( 366 bp fragment) and Figure 6 (779bp fragment). Forty-two of the 45 returned results could be included in this analysis. Three participants either did not report a result of the results were devidently based on an error in interpretation of length in gel evaluation. The mean value (XM) and standard deviation (SD) of all length determinations are illustrated in each figure. The fragment lengths defined by the majority of the participants were too high. The absolute specifications of the $366 \mathrm{bp}$ fragments (SD $\pm 23.4 \mathrm{bp}$ ) were generally more accurate than in the case of the $779 \mathrm{bp}$ fragment (SD $\pm 56.8 \mathrm{bp}$ ), although the percentage of errors was very similar (6.4\% and $7.3 \%$, respectively).

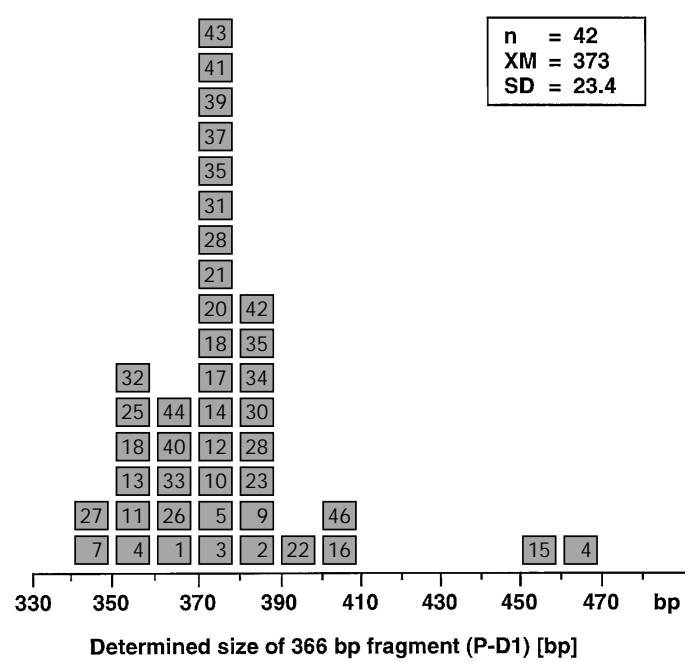

Fig. 5 Distribution of the fragment length determination of the $366 \mathrm{bp}$ product as determined by participants. Insert shows the analysis of results from 42 participating laboratories.

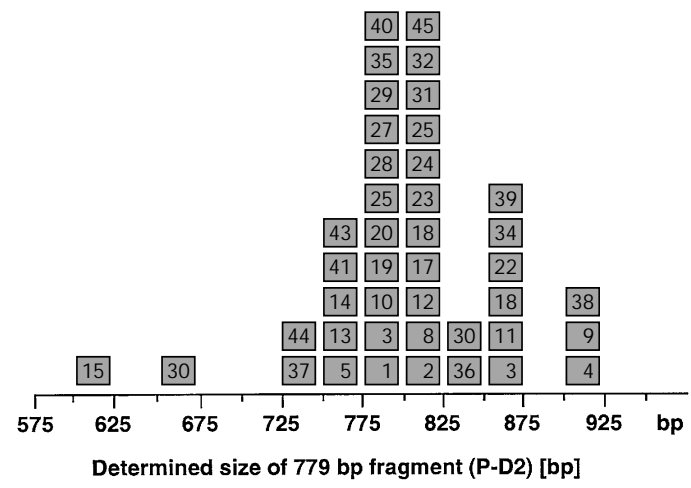

Fig. 6 Distribution of the fragment length determination of the $779 \mathrm{bp}$ product as determined by participants. Insert shows the analysis of results from 42 participating laboratories.

\section{Conclusions}

In the first external quality assessment trial for molecular genetics procedures performed by the Reference Institute of Bioanalysis of the German Society of Clinical Chemistry, most of the participants obtained comparable results. The discrepancies that are observed in some cases allow some conclusions regarding the quality of reagents and/or analytical systems used. This is a pilot study, which is an important step for the future development of better external quality assessment procedures in this field. 


\section{References}

1. Braun A, Kofler A, Morawietz S, Cleve H. Sequence and organization of the human vitamine $\mathrm{D}$-binding proteine gene. Biochim Biophys Acta 1993; 1216:385-94.

2. Braun A, Kuhnle U, Cleve H. Die Genetik der menschlichen Geschlechtsdetermination und ihre Störungen. Naturwissenschaften 1994; 81:300-7.

3. Miller SA, Dykes DD, Polesky HF. A simple salting out procedure for extracting DNA from human nucleated cells. Nucleic Acids Res 1988; 16:1215.

4. Nollau P, Moser C, Wagener C. Isolation of DNA from stool and bodily fluids for PCR amplification. Biotechniques 1996; 20:784-8.

5. Sambrook J , Fritsch EF, Maniatis T. Molecular cloning: a laboratory manual. 2nd ed. Cold Spring Harbor, NY: Cold Spring Harbor Laboratory, 1989.

6. Kunkel LM, Smith KD, Boyer SH, Borgaonkar DS, Wachtel $\mathrm{SS}$, Miller OJ , et al. Analysis of human Y-chromosome-specific reiterated DNA in chromosome variants. Proc Natl Acad Sci USA 1977; 74:1245.
7. Rolfs A, Schuller I, Finck U, Weber-Rolfs I. PCR: clinical diagnostics and research. Berlin, Heidelberg, New York: Springer-Verlag 1992:68-78.

8. Olerup $O$, Zetterquist $H$. HLA-DR typing by PCR amplification with sequence-specific primers (PCR-SSP) in 2 hours: an alternative to serological DR typing in clinical practice including donor-recipient matching in cadaveric transplantation. Tissues Antigens 1992; 39:225-35.

9. Higuchi R. Simple and rapid preparation of samples for PCR. In: Ehrlich HA, editor. PCR technology. New York: Stockton Press, 1989; 31-8.

\section{Received 21J uly 1997; accepted 19 February 1998}

Prof. Dr. med. Christoph Wagener, Abteilung für Klinische Chemie, Universitätskrankenhaus Eppendorf, Martinistr. 52, D-20251 Hamburg, Germany

Tel.: +49-40-47 17-2981, Fax: +49-40-47 17-4621

E-mail:wagener@uke.-uni-hamburg.de 